\title{
On Kerr-Schild spacetimes in higher dimensions ${ }^{1}$
}

\author{
M. Ortaggio, V. Pravda and A. Pravdová \\ Institute of Mathematics, Academy of Sciences of the Czech Republic \\ Žitná 25, 11567 Prague 1, Czech Republic
}

\begin{abstract}
We summarize main properties of vacuum Kerr-Schild spacetimes in higher dimensions.
Keywords: Higher dimensional gravity; Kerr-Schild geometry

PACS: 04.50.+h, 04.20.-q, 04.20.Cv
\end{abstract}

\section{INTRODUCTION}

Kerr-Schild (KS) spacetimes [1] possess a rare property of being physically important and yet mathematically tractable. In $n=4$ dimensions they contain important exact vacuum solutions such as the Kerr metric and pp-waves. Vacuum KS spacetimes are algebraically special and thus, because of the Goldberg-Sachs theorem, the KS null congruence is geodetic and shearfree. Thanks to this and to the Kerr theorem [2, 3, 4], the general $n=4$ vacuum KS solution is in fact known [1, 2, 5, 6]. In arbitrary higher dimensions, the KS ansatz led to the discovery of rotating vacuum black holes [7]. Here we will study the general class of $n>4 \mathrm{KS}$ spacetimes [8].

Note that in $n>4$ gravity there is no unique generalization of the shearfree condition [9, 10, 11, 12, 13, 14] and, a fortiori, it is not obvious how to extend the Goldberg-Sachs theorem. In fact, it has been pointed out that this can not be done in the most direct way [7, 15, 16, 17, 18]. Our results below will suggest a possible weak generalization of the shearfree condition, and a partial extension of the Goldberg-Sachs theorem to $n>4$ (limited to KS solutions). It is worth mentioning that an $n>4$ extension of the Robinson theorem has been proven in even dimensions [10] assuming a generalization of the shearfree condition (see also [11, 12, 13, 14]) different from ours. The relation to our work will be discussed elsewhere. Let us also recall that properties of KS transformations in arbitrary dimensions have been studied in [19]. This does not overlap significantly with our contribution.

\section{GEOMETRIC OPTICS AND ALGEBRAICAL PROPERTIES}

By definition, Kerr-Schild spacetimes in $n \geq 4$ dimensions are metrics of the form

$$
g_{a b}=\eta_{a b}-2 \mathscr{H} k_{a} k_{b}
$$

\footnotetext{
${ }^{1}$ Proceedings of the Spanish Relativity Meeting 2008, Salamanca, September 15-19, 2008 (http://www.usal.es/ere2008/)
} 
where $\eta_{a b}=\operatorname{diag}(-1,1, \ldots, 1)$ is the Minkowski metric, $\mathscr{H}$ a scalar function and $k_{a}$ a 1 -form that is assumed to be null with respect to $\eta_{a b}$, i.e. $\eta^{a b} k_{a} k_{b}=0$ ( $\eta^{a b}$ is defined as the inverse of $\eta_{a b}$ ). Hence $k^{a} \equiv \eta^{a b} k_{b}=g^{a b} k_{b}$, so that $k^{a}$ is null also with respect to $g_{a b}$.

It can be shown that optical properties of $k^{a}$ in the full KS geometry $g_{a b}$ are inherited from the flat background spacetime $\eta^{a b}$. More specifically, the matrix $L_{i j}$ is the same in both spacetimes, i.e

$$
L_{i j} \equiv k_{a ; b} m^{(i) a} m^{(j) b}=k_{a, b} m^{(i) a} m^{(j) b},
$$

and so are the optical scalars expansion $\theta=L_{i i} /(n-2)$, shear $\sigma^{2}=L_{(i j)} L_{(i j)}-(n-2) \theta^{2}$ and twist $\omega^{2}=L_{[i j]} L_{[i j]}$. Furthermore, $k^{a}$ is geodetic with respect to $g_{a b}$ iff it is geodetic in $\eta^{a b}$. This geometric condition on $k^{a}$ turns out to constraint the possible form of the energy-momentum tensor $T_{a b}$ compatible with $g_{a b}$, i.e.

Proposition 1 The null vector $k^{a}$ in the KS metric (1) is geodetic iff $T_{a b} k^{a} k^{b}=0$.

Note that this condition is satisfied, e.g., in the case of vacuum spacetimes, also with a possible cosmological constant, or in the presence of matter fields aligned with the KS vector $k^{a}$, such as an aligned Maxwell field or aligned pure radiation.

Further computation constrains also the algebraic type of the Weyl tensor

Proposition 2 If $k^{a}$ is geodetic, KS spacetimes (1) are of type II (or more special).

From the results of [18], it follows that static and (a specific subclass of) stationary spacetimes belonging to the KS class are necessarily of type $\mathrm{D}$, and $k^{a}$ is a multiple WAND. As a consequence, Myers-Perry black holes must be of type D (cf. also [20]). By contrast, black rings do not admit a KS representation, since they are of type $I_{i}[21]$.

\section{VACUUM SOLUTIONS}

In the rest of the paper we will focus on vacuum solutions and, by Proposition $1, k^{a}$ will thus be geodetic. Some of the vacuum equations are remarkably simple thanks to the metric ansatz (1). In particular, imposing $R_{i j}=0$ we obtain

$$
(D \ln \mathscr{H}) S_{i j}=L_{i k} L_{j k}-(n-2) \theta S_{i j}
$$

(where $D \equiv k^{a} \nabla_{a}$ ) and its contraction with $\delta^{i j}$ gives

$$
(n-2) \theta(D \ln \mathscr{H})=\sigma^{2}+\omega^{2}-(n-2)(n-3) \theta^{2} .
$$

The latter involves $\mathscr{H}$ only when $\theta \neq 0$, so that KS spacetimes naturally split into two families with either $\theta=0$ (non-expanding) and $\theta \neq 0$ (expanding).

\section{Non-expanding solutions}

It turns out that the vacuum KS subfamily $\theta=0$ can be integrated in full generality. Our analysis, combined with the results of [22] (where all vacuum Kundt type $\mathrm{N}$ 
solutions have been given) shows that in arbitrary $n \geq 4$ dimensions

Proposition 3 The subfamily of Kerr-Schild vacuum spacetimes with a non-expanding $K S$ congruence $k^{a}$ coincides with the class of vacuum Kundt solutions of type $N$.

A simple explicit example of non-expanding KS solutions is given by $p p$-waves of type $\mathrm{N}$ (cf. [22] and references therein). But note that, as opposed to the case $n=4$, for $n>4$ not all $p p$-waves fall into the KS class, and in fact they can also be of Weyl types different from $\mathrm{N}$ (see [8] for details).

\section{Expanding solutions}

The subfamily of expanding solutions is more complex and contains, in particular, Myers-Perry black holes [7]. When $\theta \neq 0$, from eqs. (3) and (4) one gets

$$
L_{i k} L_{j k}=\frac{L_{l k} L_{l k}}{(n-2) \theta} S_{i j}
$$

Remarkably, this equation is independent of the function $\mathscr{H}$. It is thus a purely geometric condition on the KS null congruence $k^{a}$ in the Minkowskian "background" $\eta_{a b}$ (an optical constraint). It is important in proving further properties of expanding solutions.

\section{Optics}

First, the optical constraint implies $L L^{T}-L^{T} L=0$, i.e. $L$ is a normal matrix. Combining this with the Ricci identities [17], one can prove that there exists a "canonical" frame in which the matrix $L_{i j}$ takes a specific block-diagonal form, with a number $p$ of $2 \times 2$ blocks $\mathscr{L}_{(\mu)}$, and a single diagonal block $\tilde{\mathscr{L}}$ of dimension $(n-2-2 p) \times(n-2-2 p)$. They are given by

$$
\begin{aligned}
& \mathscr{L}_{(\mu)}=\left(\begin{array}{cc}
s_{(2 \mu)} & A_{2 \mu, 2 \mu+1} \\
-A_{2 \mu, 2 \mu+1} & s_{(2 \mu)}
\end{array}\right) \quad(\mu=1, \ldots, p), \\
& s_{(2 \mu)}=\frac{r}{r^{2}+\left(a_{(2 \mu)}^{0}\right)^{2}}, \quad A_{2 \mu, 2 \mu+1}=\frac{a_{(2 \mu)}^{0}}{r^{2}+\left(a_{(2 \mu)}^{0}\right)^{2}}, \\
& \tilde{\mathscr{L}}=\frac{1}{r} \operatorname{diag}(\underbrace{1, \ldots, 1}_{(m-2 p)}, \underbrace{0, \ldots, 0}_{(n-2-m)}),
\end{aligned}
$$

with $0 \leq 2 p \leq m \leq n-2$. (The integer $m \geq 2$ is the rank of $L_{i j}$. From now on, a superscript (or subscript) index 0 denotes quantities independent of $r$, which is an affine parameter along $k^{a}$.) The above special properties of the matrix $L_{i j}$ can be viewed as a "generalization" of the shearfree condition and considered in a weak formulation of the Goldberg-Sachs theorem in $n>4$ dimensions, restricted to KS solutions [8]. 


\section{Singularities}

Together with the Einstein equation (4), eqs. (6)-(8) in turn enable one to fix also the $r$-dependence of $\mathscr{H}$, i.e.

$$
\mathscr{H}=\frac{\mathscr{H}_{0}}{r^{m-2 p-1}} \prod_{\mu=1}^{p} \frac{1}{r^{2}+\left(a_{(2 \mu)}^{0}\right)^{2}} .
$$

The above functional dependence suggests there may be singularities at $r=0$, at least for $2 p \neq m$ ( $m$ even) and $2 p \neq m-1$ ( $m$ odd). This singular behaviour can indeed be confirmed by examining the Kretschmann scalar. Singularities may also be present in the special cases $2 p=m$ and $2 p=m-1$ at "special points" with $r=0$ and where some of the $a_{(2 \mu)}^{0}$ vanish. See [8] for more details and [7] for a thorough discussion of singularities in the special case of rotating black hole spacetimes.

$$
\text { Weyl type }
$$

Along with the Bianchi identities [16], the optical contraint also imply that expanding vacuum KS solutions can not be of the type III or $\mathrm{N}$, so that in arbitrary dimension $n \geq 4$

Proposition 4 Kerr-Schild vacuum spacetimes with an expanding KS congruence $k^{a}$ are of algebraic type II or D.

\section{ACKNOWLEDGMENTS}

The authors acknowledge support from research plan No AV0Z10190503 and research grant KJB100190702. M.O. also thanks the conference organizers and the European Network of Theoretical Astroparticle Physics ILIAS/N6 under contract number RII3CT-2004-506222 for financial support to his participation to the Spanish Relativity Meeting 2008.

\section{REFERENCES}

1. R. P. Kerr, and A. Schild, Proc. Symp. Appl. Math. 17, 199-209 (1965).

2. G. C. Debney, R. P. Kerr, and A. Schild, J. Math. Phys. 10, 1842-1854 (1969).

3. R. Penrose, J. Math. Phys. 8, 345-366 (1967).

4. D. Cox, and E. J. Flaherty Jr., Commun. Math. Phys. 47, 75-79 (1976).

5. H. K. Urbantke, Acta Phys. Aust. 35, 396-397 (1972).

6. H. Stephani, D. Kramer, M. MacCallum, C. Hoenselaers, and E. Herlt, Exact Solutions of Einstein's Field Equations, Cambridge University Press, Cambridge, 2003, second edn.

7. R. C. Myers, and M. J. Perry, Ann. Phys. (N.Y.) 172, 304-347 (1986).

8. M. Ortaggio, V. Pravda, and A. Pravdová, Class. Quantum Grav. 26, 025008 (2009).

9. I. Robinson, and A. Trautman, J. Math. Phys. 24, 1425-1429 (1983).

10. L. P. Hughston, and L. J. Mason, Class. Quantum Grav. 5, 275-285 (1988).

11. P. Nurowski, and A. Trautman, Differential Geom. Appl. 17, 175-195 (2002). 
12. A. Trautman, Class. Quantum Grav. 19, R1-R10 (2002).

13. A. Trautman, Int. J. Mod. Phys. A 17, 2735-2737 (2002).

14. L. J. Mason, and A. Taghavi-Chabert (2008), arXiv:0805.3756v1 [math.DG]

15. V. P. Frolov, and D. Stojković, Phys. Rev. D 68, 064011 (2003).

16. V. Pravda, A. Pravdová, A. Coley, and R. Milson, Class. Quantum Grav. 21, 2873-2897 (2004), see also V. Pravda, A. Pravdová, A. Coley and R. Milson Class. Quantum Grav. 24 (2007) 1691 (corrigendum).

17. M. Ortaggio, V. Pravda, and A. Pravdová, Class. Quantum Grav. 24, 1657-1664 (2007).

18. V. Pravda, A. Pravdová, and M. Ortaggio, Class. Quantum Grav. 24, 4407-4428 (2007).

19. B. Coll, S. R. Hildebrandt, and J. M. M. Senovilla, Gen. Rel. Grav. 33, 649-670 (2001).

20. N. Hamamoto, T. Houri, T. Oota, and Y. Yasui, J. Phys. A 40, F177-F184 (2007).

21. V. Pravda, and A. Pravdová, Gen. Rel. Grav. 37, 1277-1287 (2005).

22. A. Coley, A. Fuster, S. Hervik, and N. Pelavas, Class. Quantum Grav. 23, 7431-7444 (2006). 